\title{
“ANÁLISIS DEA DE LA EFICIENCIA-PAIS EN LA APORTACIÓN DE LA PRODUCCIÓN AGROALIMENTARIA ECOLÓGICA EN LA UNIÓN EUROPEA 27”
}

\author{
Antonio Colom Gorgues*a, Rosa M. Florensa Guiu ${ }^{\text {b }}$ Eduardo Cristóbal Fransic \\ a) Escuela Técnica Superior de Ingeniería Agraria, Departamento de Administración de Empresas, \\ Universidad de Lleida; email: antonio_colom@hotmail.com ; ORCID: 0000-0001-7129-2539 \\ b) Escuela Superior Politécnica, Departamento de Administración de Empresas, Universidad de Lleida; \\ email: rosa.florensa@udl.cat ; ORCID:0000-0002-1791-3266 \\ c) Facultad de Derecho, Economía y Turismo, Departamento de Administración de Empresas, Universidad \\ de Lleida; email: eduard.cristobal@udl.cat ;ORCID: 0000-0003-1795-6263
}

\section{Resumen:}

La UE plantea que la producción ecológica es un sistema general de gestión agraria y producción de alimentos que combina las mejores prácticas en materia de medio ambiente y clima, un elevado nivel de biodiversidad, la conservación de los recursos naturales y la aplicación de normas exigentes sobre bienestar animal y sobre producción sostenible de cara a un creciente número de consumidores, de productos obtenidos a partir de sustancias y procesos naturales. Se conoce que la producción ecológica forma parte de regímenes de calidad de los productos agroalimentarios de la UE, junto con DOP-IGO-ETG, de conformidad con el Reglamento (UE) 1151/2012.

El objeto de esta comunicación es resumir una investigación basada en la metodología de Data Envelopment Analysis (DEA) para evaluar técnicamente el nivel de eficiencia-país en la aportación de la producción agroalimentaria ecológica por cada país de la Unión Europea 27. España lidera la superficie cultivada de producción agrícola ecológica, aunque mantiene un lugar discreto en cuanto a consumo. Los resultados de esta investigación permitirán conocer el ranking de los países que más eficientemente se preocupan y se comportan en dicho tipo de producción sostenible, considerando los inputs y outputs que se relacionan con dichas prácticas productivas.

Palabras Clave: Producción Ecológica, Producción Sostenible, Data Envelopment Analysis, Eficiencia Técnica, Eficiencia-País.

\section{Introducción. Objetivo de la comunicación}

Como es sabido, la Unión Europea como bloque socioeconómico propugna la atención, actuaciones y seguimiento de los Objetivos de Desarrollo Sostenible (ODS) promovidos y planteados por Naciones Unidas en su Agenda 2030. El elemento clave, que es el Desarrollo Sostenible, tiene mucho que ver con la cadena alimentaria, es decir, con la agricultura, la ganadería, los recursos naturales y la producción y distribución de alimentos.

Por un lado, y en relación con dicha sostenibilidad, la Unión Europea declara y tiene en cuenta que la producción ecológica es un sistema general de gestión agraria y producción de alimentos que combina las mejores prácticas respecto el medio ambiente y clima, un elevado nivel de protección a la biodiversidad, la conservación de los recursos naturales y la aplicación de normas exigentes sobre el bienestar animal y sobre la producción sostenible de cara a un creciente número de consumidores, de productos obtenidos a partir de sustancias y procesos naturales. Por otro lado, se sabe que la producción ecológica forma parte de los regímenes de calidad de los productos agroalimentarios de la UE, junto con DOP-IGO-ETG, de conformidad con el Reglamento (UE) 1151/2012.

El objetivo de esta comunicación es resumir, en términos de eficiencia-país, la producción sostenible de los 27 países de la Unión Europea determinando el ranking de los países que más eficientemente se preocupan y se comportan en dicho tipo de producción sostenible, considerando los inputs y outputs que se relacionan con dichas prácticas productivas.

\section{Esquema metodológico.}

La metodología prevista para el proceso investigador necesario para realizar esta comunicación se esquematiza:

1. Búsqueda de datos sobre producción ecológica en la Unión Europea-27 a través de Eurostat, FiBLIFOAM, y obtención de los inputs y outputs decididos en el modelo DEA. Al mismo tiempo contextualización teórica del cálculo de la eficiencia a través del Data Envelopment Analysis (DEA).

2. Aplicación del DEA a los inputs y outputs del modelo para el cálculo de la Eficiencia-país en la UE27 utilizando la Hoja de Cálculo Excel con macros a través de Solver.

3. Presentación de resultados, análisis de estos, y ranking de países por su eficiencia.

4. Conclusiones. 


\section{Data Envelopment Analysis (Dea). Aplicación del modelo Dea}

El científico pionero de la Eficiencia en procesos productivos, y su medición, fue Farell (1957) que consideró la Eficiencia Técnica y la Asignativa. La primera se refiere a la habilidad de una empresa para obtener el máximo nivel de producción dado un conjunto de cantidades de inputs o, a partir de un nivel dado de producto, obtenerlo con la menor combinación de cantidades de inputs. La segunda muestra la habilidad de una empresa para usar los inputs en proporciones óptimas, dados los precios de éstos, y obtener un determinado nivel de producción con el menor coste o, para determinado nivel de costes, obtener la máxima cantidad de producto. Ambas medidas, combinadas, definen la medida de la Eficiencia Económica. En su acepción maximalista, Farrell planteó que las empresas que estuvieran en la frontera (maximizando la producción dado un conjunto de inputs; o minimizando la combinación de inputs para obtener un nivel productivo dado) gozarían del rango máximo de Eficiencia del 100\% (o Eficiencia 1), mientras que las empresas que no llegaran a dicha frontera, relativamente serían Ineficientes con grado de medida $<100 \%$ (o Eficiencia < 1).

A partir de la segunda mitad y finales del siglo XX, los investigadores crearon y desarrollaron diversos métodos para medir la eficiencia, algunos paramétricos y otros no paramétricos. Destacan por sus numerosas aplicaciones dos metodologías: la estimación de Fronteras Probabilísticas o Estocásticas; y la aplicación del Análisis Envolvente de Datos (en términos anglosajones, Data Envelopment Analysis o DEA). Este segundo método, que goza de gran aceptación y grado de utilidad, es el que se aplica aquí.

DEA utiliza un conjunto de inputs y un conjunto de outputs utilizados y obtenidos por una Unidad de Gestión de Datos o de Toma de Decisiones (originalmente, DMU o Data Management Unit), y aplica la programación lineal para, a través de la envolvente de dichos datos, discernir el grado o medida de eficiencia de las DMU's. Inicialmente el modelo DEA fue propuesto por Rhodes (1978) en su Tesis Doctoral, y posteriormente publicado por Charnes, Cooper y Rhodes (1978) y se trataba de un modelo fraccional (ver gráfica 1) con la búsqueda de la solución mediante programación lineal.

Gráfica 1. Modelo DEA original de Rhodes (1978) y modelo posterior de Charnes et alt (1978)

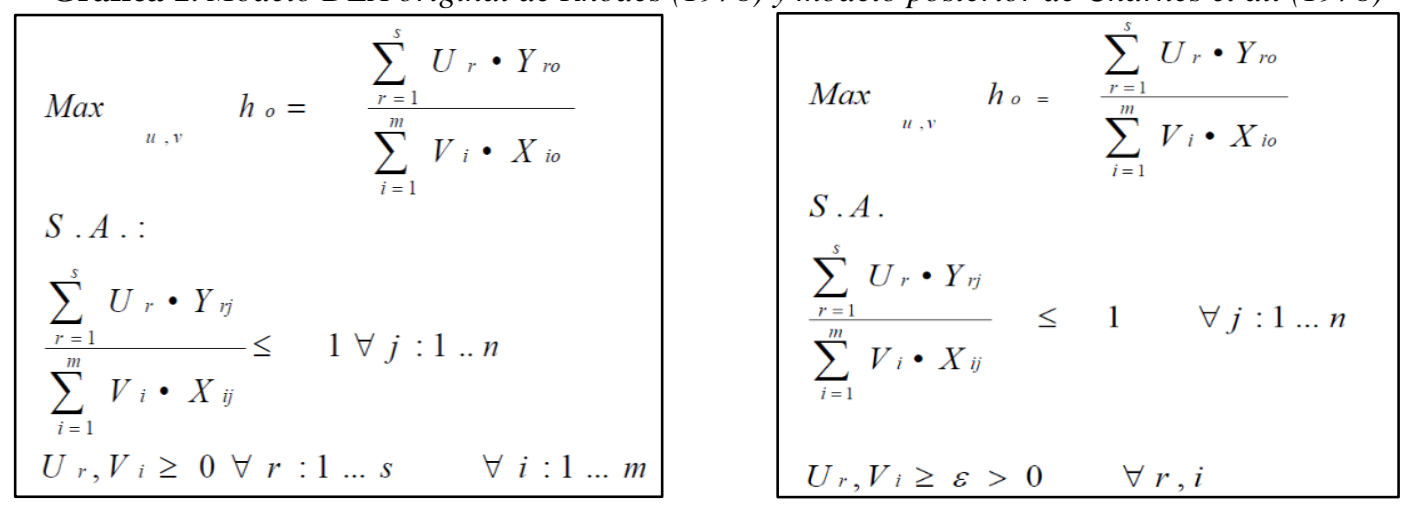

Fuente: Elaboración propia

... donde:

$\mathrm{h}_{0}$ : Función Objetivo. Medida de la Eficiencia.

$Y_{\mathrm{rj}}$ : Output i-ésimo (hay “s” outputs) de la DMU j-ésima (hay “n” DMU's).

$\mathrm{X}_{\mathrm{ij}}$ : Input i-ésimo (hay " $\mathrm{m}$ " inputs) de la DMU j-ésima.

$\mathrm{V}_{\mathrm{i}}, \mathrm{U}_{\mathrm{r}}$ : Ponderaciones de inputs y outputs respectivamente (soluciones del programa).

$\varepsilon$ : Número real positivo y pequeño (usualmente, en cálculos empíricos, $10^{-6}$

Para que el cálculo fuera más operativo se linealizó el modelo, llegando a la expresión de la figura 2.

Gráfica 2. Modelo DEA de Charnes et alt (1978) linealizado

\begin{tabular}{l} 
Max ${ }_{u, v}=\sum_{r=1}^{s} U_{r} \cdot Y_{r o}$ \\
$S . A$. \\
$\sum_{i=1}^{m} V_{i} \cdot X_{i o}=1$ \\
$\sum_{r=1}^{s} U_{r} \cdot Y_{r j}-\sum_{i=1}^{m} V_{i} \cdot X_{i j} \leq 0 \quad \forall j: 1 \ldots n$ \\
$U_{r,}, V_{i} \geq \varepsilon>0 \quad \forall r, i$. \\
\hline
\end{tabular}

Fuente: Elaboración propia 
Este modelo anterior, que en reconocimiento a sus autores se denominó CCR (Charnes, Cooper, Rhodes), fue primeramente aplicado asumiendo Rendimientos Constantes a Escala (CRS, en inglés) de las DMU. Posteriormente, Banker, Charnes y Cooper (1984) propusieron el modelo BCC que asumía Rendimientos Variables a Escala (VRS). Las DMU que obtuvieran un índice " $\mathrm{h}_{0}=1$ " serían globalmente eficientes, mientras que aquellas que obtuvieran " $\mathrm{h}_{0}<1$ " serían ineficientes. La puntuación de VRS sólo mide la eficiencia técnica pura. Sin embargo, para fines comparativos, se pueden calcular tasas de rendimientos constantes de escala, que comprende una combinación no aditiva de eficiencias técnicas puras y de escala. La relación entre el índice de eficiencia global (puntuación CRS) y el índice de eficiencia técnica pura (puntuación VRS) proporciona una medida de la eficiencia de escala.

En aplicación a este caso, se han considerado para cada uno de los 27 países de la UE, los INPUTS: 1. Superficie Ha de cultivo ecológico; 2. Número de Explotaciones solo ecológicas; 3. Número de Explotaciones parciales ecológicas; y 4. Cabezas Ganado Bovino ecológico (carne+leche); y por otro lado los OUTPUTS: 1. Cifra de Ventas de Productos Ecológicos en Retail, Millones €; y 2. Ratio de Standard Output (SO) en $€$ / Unidad Trabajo Anual. Estos datos se muestran en el cuadro 1.

Cuadro 1. Conjunto de Inputs (4) y Outputs (2) considerados en el modelo DEA

\begin{tabular}{|c|c|c|c|c|c|c|c|}
\hline $\mathbf{N}^{0}$ & DMU: País de la UE-27 & \begin{tabular}{|l|} 
Input1: Superficie \\
Ha de cultivo \\
ecológico
\end{tabular} & \begin{tabular}{|l|} 
Input2: Número de \\
Explotaciones \\
solo ecológicas \\
\end{tabular} & $\begin{array}{l}\text { Input3: Número de } \\
\text { Explotaciones } \\
\text { parciales ecológicas }\end{array}$ & $\begin{array}{l}\text { Input4: Cabezas } \\
\text { Ganado Bovino } \\
\text { ecológico }\end{array}$ & \begin{tabular}{|l|} 
Output1: Cifra Ventas \\
Productos Ecológicos \\
en Retail, Millones $€$
\end{tabular} & $\begin{array}{l}\text { Output2: Ratio de } \\
\text { Standard Output (SO) en } \\
€ \text { / Udad Trabajo Anual } \\
\end{array}$ \\
\hline 1 & Bélgica & $\begin{array}{r}93.119 \\
\end{array}$ & $\begin{array}{r}1.000 \\
\end{array}$ & 490 & 130.139 & 979 & $96.585,70$ \\
\hline 2 & Bulgaria & 117.779 & 2.160 & 2.150 & 12.435 & 30 & $12.185,60$ \\
\hline 3 & Rep. Checa & 535.185 & 2.930 & 50 & 270.157 & 164 & $31.310,50$ \\
\hline 4 & Dinamarca & 285.526 & 1.420 & 1.150 & 302.511 & 1.979 & $170.593,10$ \\
\hline 5 & Alemania & 1.290 .839 & 19.800 & 490 & 1.097 .284 & 11.970 & $60.746,20$ \\
\hline 6 & Estonia & 220.737 & 1.570 & 100 & 44.125 & 55 & $42.384,10$ \\
\hline 7 & Irlanda & 73.952 & 200 & 1.380 & 69.501 & 206 & $18.950,20$ \\
\hline 8 & Grecia & 528.752 & 4.580 & 8.080 & 159.565 & 66 & $20.197,50$ \\
\hline 9 & España & 2.354 .916 & 14.550 & 13.610 & 226.215 & 2.133 & $35.921,40$ \\
\hline 10 & Francia & 2.240 .797 & 21.620 & 6.630 & 990.499 & 11.295 & $52.839,40$ \\
\hline 11 & Croacia & $\begin{array}{l}108.127 \\
\end{array}$ & 1.250 & 2.270 & 21.915 & 99 & $17.984,30$ \\
\hline 12 & Italia & 1.993 .225 & 56.060 & 9.220 & 469.207 & 3.625 & $53.893,80$ \\
\hline 13 & Chipre & 6.240 & 270 & 300 & 1.327 & 2 & $25.964,70$ \\
\hline 14 & Letonia & 289.796 & 420 & 3.250 & 117.158 & 51 & $14.526,30$ \\
\hline 15 & Lithuania & 242.118 & 890 & 1.580 & 72.509 & 51 & $29.866,80$ \\
\hline 16 & Luxemburgo & 5.814 & 83 & 50 & 5.531 & 160 & 0,00 \\
\hline 17 & Hungría & 303.190 & 780 & 1.730 & 28.207 & 30 & $26.011,30$ \\
\hline 18 & Malta & 55 & 20 & 15 & 0 & 0 & 0,00 \\
\hline 19 & Holanda & 68.068 & 970 & 400 & 109.719 & 1.211 & $114.114,00$ \\
\hline 20 & Austria & 671.703 & 21.990 & 520 & 536.064 & 1.870 & $42.052,90$ \\
\hline 21 & \begin{tabular}{|l|} 
Polonia \\
\end{tabular} & 507.637 & 2.130 & 17.500 & 41.169 & 314 & $22.426,80$ \\
\hline 22 & Portugal & 293.213 & 740 & 1.310 & 95.308 & 21 & $19.186,30$ \\
\hline 23 & Rumania & 395.228 & 630 & 1.750 & 33.240 & 41 & $29.309,60$ \\
\hline 24 & Eslovenia & 49.638 & 2.910 & 680 & 37.126 & 49 & $13.944,30$ \\
\hline 25 & Eslovaquia & 197.565 & 340 & 90 & 67.191 & 4 & $30.780,50$ \\
\hline 26 & Finlandia & 306.484 & 3.040 & 1.190 & 86.197 & 368 & $33.216,40$ \\
\hline 27 & Suecia & 613.964 & 4.040 & 1.610 & 389.989 & 2.144 & $98.049,60$ \\
\hline
\end{tabular}

Fuente: Elaboración propia con datos del año 2019 obtenidos de Eurostat y FiBL-IFOAM, 2021

A continuación se ha aplicado este modelo DEA CCR a los datos anteriores, considerando pues los 4 Inputs o Entradas y los dos Outputs o Salidas referidos.

\section{RESULTADOS, ANÁLISIS Y DIAGNÓSTICO}

Se ha aplicado en la Hoja de Cálculo Excel el DEA Spreadsheet Solver, versión 1.1 (6 de marzo de 2021), diseñado en formato de programa informático libre por el Dr. Güneş Erdogan, de la Management School de la University of Bath (UK).

Gráfica 3. Datos de partida para la programación lineal del DEA

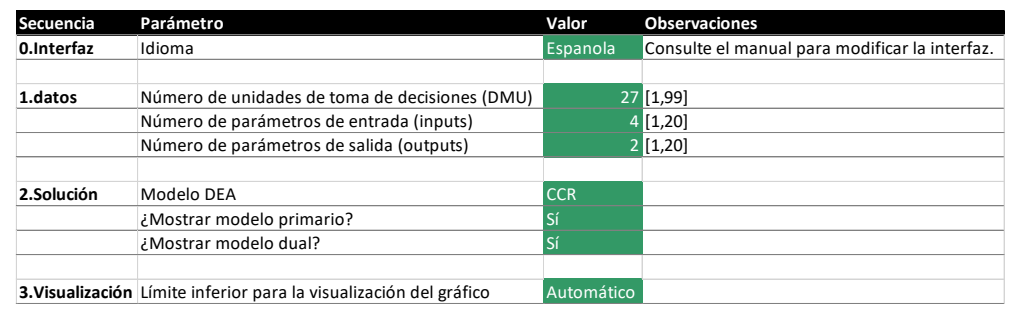

Fuente: Aplicación propia en DEA Spreadsheet Solver v. 1.1

Se ha procedido a entrar los primeros datos según se refleja en la gráfica 3 y se han seguido las instrucciones para poner en marcha el programa lineal que se ha ejecutado a través de una macro que ha activado el módulo Solver de Excel.

En segundo lugar se ha activado la entrada de datos, la hoja de soluciones y la hoja de visualización de resultados, tal como se observa en la gráfica 4 . 
Finalmente se ha pulsado el botón 4. Solucionador, para poner en marcha la macro que acciona el módulo de Solver de la Hoja de Cálculo Excel y así acceder al Análisis Envolvente de Datos CCR, cuyos resultados se muestran en la gráfica 5 .

Gráfica 4. Aplicación del DEA Spreadsheet Solver en nuestro caso

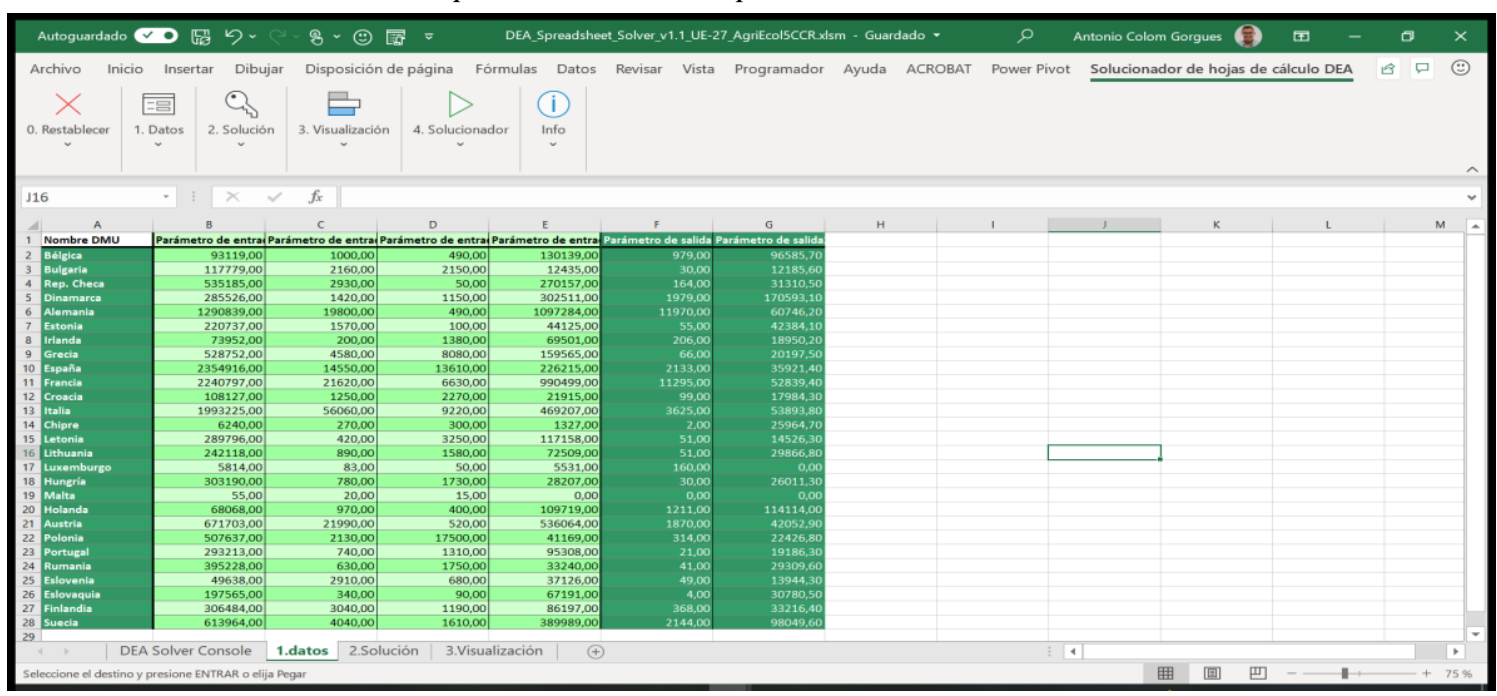

Fuente: Aplicación propia en DEA Spreadsheet Solver v. 1.1

Gráfica 5. Resultados obtenidos de la aplicación del DEA a los 27 países de la Unión Europea

\begin{tabular}{l|r|} 
Nombre DMu & $\begin{array}{r}\text { Puntuación de } \\
\text { eficiencia }\end{array}$ \\
\hline Bélgica & $81,81 \%$ \\
Bulgaria & $13,09 \%$ \\
Rep. Checa & $100,00 \%$ \\
Dinamarca & $100,00 \%$ \\
Alemania & $100,00 \%$ \\
Estonia & $100,00 \%$ \\
Irlanda & $78,87 \%$ \\
Grecia & $4,32 \%$ \\
España & $33,36 \%$ \\
Francia & $48,75 \%$ \\
Croacia & $19,59 \%$ \\
Italia & $27,26 \%$ \\
Chipre & $100,00 \%$ \\
Letonia & $28,79 \%$ \\
Lithuania & $30,13 \%$ \\
Luxemburgo & $100,00 \%$ \\
Hungría & $32,58 \%$ \\
Malta & $0,00 \%$ \\
Holanda & $100,00 \%$ \\
Austria & $33,69 \%$ \\
Polonia & $29,00 \%$ \\
Portugal & $21,97 \%$ \\
Rumania & $44,03 \%$ \\
Eslovenia & $16,58 \%$ \\
Eslovaquia & $100,00 \%$ \\
Finlandia & $26,70 \%$ \\
Suecia & $39,75 \%$
\end{tabular}

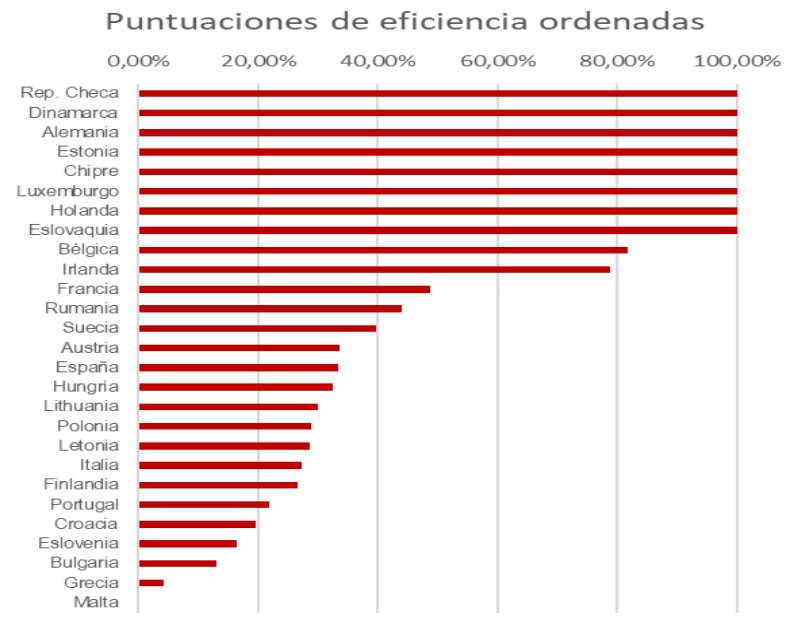

Fuente: Aplicación propia en DEA Spreadsheet Solver v. 1.1

\section{CONCLUSIONES}

Se destaca la Eficiencia Global de países como Alemania, Dinamarca y Holanda, que aunque no lideran los inputs de producción ecológica (excepto Alemania que si lidera el número de cabezas bovinas ecológicas), tienen una gran distribución y consumo de productos ecológicos. España, que se encuentra en el nivel de eficiencia de países como Austria, Hungría y Lithuania, no sabe cómo impulsar la distribución y el consumo ecológico en nuestro país. No obstante, la tendencia al crecimiento de consumo de estos productos vaticina una mejora de la situación del sector, que a veces está forzado a aceptar precios parecidos a los productos convencionales, a sabiendas que los costes de producción son claramente más elevados.

\section{BIBLIOGRAFIA}

Banker R., Charnes A., Cooper W.W. (1984). "Some models for estimating technical and scale inefficiencies in Data Envelopment Analysis". Management Science, vol. 30, n. ${ }^{\circ}$ 9, pp 1078-1092.

Cooper W.W., Seiford L.M., Tone K. (1999). Data Envelopment Analysis A Comprehensive Text with Models, Applications, References and DEA-Solver Software. Kluver Nijhoff Publishing, Boston (USA).

Charnes A., Cooper W.W., Rhodes E. (1978). "Measurement the efficiency of decision making units". European Journal of Operational Research, vol. 2, pp 429-444. 
Charnes A., Cooper W.W., Lewin A.Y., Seiford L.M. (1995). Data Envelopment Analysis: Theory, Methodology and Applications. Kluver Nijhoff Publishing, Boston (USA).

Farrell M. (1957). "The measurement of productive efficiency". Journal of the Royal Statistical Society (Series A), 120, part. III, pp 253-290. 